\title{
Gadda : grades et dégâts. Chronique d'une recherche du sens
}

\section{Christophe Mileschi}

\section{(2) OpenEdition}

\section{Journals}

Édition électronique

URL : http://journals.openedition.org/cei/235

DOI : $10.4000 /$ cei.235

ISSN : 2260-779X

\section{Éditeur}

UGA Éditions/Université Grenoble Alpes

\section{Édition imprimée}

Date de publication : 15 novembre 2004

Pagination : 73-89

ISBN : 978-2-84310-057-4

ISSN : 1770-9571

\section{Référence électronique}

Christophe Mileschi, «Gadda : grades et dégâts. Chronique d'une recherche du sens », Cahiers d'études italiennes [En ligne], 1 | 2004, mis en ligne le 15 mai 2006, consulté le 27 mars 2021. URL : http:// journals.openedition.org/cei/235; DOI : https://doi.org/10.4000/cei.235 


\title{
GADDA \\ GRADES ET DÉGÂTS \\ CHRONIQUE D'UNE RECHERCHE DU SENS
}

\author{
Christophe Mileschi \\ Université Stendhal-Grenoble 3
}

ASPIDO.
Il morso di questo animale non ha rimedio se non di
subito tagliare le parti morse.
Leonardo da Vinci.

Ma découverte de Gadda remonte au mois de juin 1994, lorsque je sus qu'il m'incomberait à la rentrée universitaire suivante de faire cours sur son œuvre, inscrite au programme du CAPES et de l'agrégation de la session 1995. Jusque là, n'ayant lu que quelques textes ou bribes de textes épars, j'avais de Gadda la vision - réductrice, comme on verra - d'un écrivain attaché à l'expérimentation linguistique comme fin en soi, ayant laissé une œuvre d'accès difficile, et, en somme, se situant plutôt en dehors de l'histoire - ou qu'on ne pouvait escompter comprendre en regardant du côté de l'histoire. C'est couramment l'idée que véhicule, aujourd'hui encore, une certaine critique littéraire (que, pour simplifier, on peut appeler "critique narratologique»): Gadda serait une sorte de génie isolé, un cas à lui tout seul, un des très rares auteurs italiens dont l'œuvre ne peut être intimement saisie et entendue qu'à la condition de mettre en œuvre un savant appareillage de décryptage - structuraliste, ou post-structuraliste, ou psychanalytique, en tout cas fort éloigné de ce que les lectures naïves permettent d'entendre. Un écrivain hors du commun, au sens littéral de cette expression, dont la complexité de l'œuvre prouve que la littérature est sans commune mesure avec la vie, et que la science qui tente de la déchiffrer (la critique) est une discipline cognitive séparée, avec ses équations, son langage spécifique, ses procédures propres. Je ne dis pas que c'est ce que dit toute la critique gaddienne, ni même toute la critique 
«narratologique», mais, simplement, que c'est ce que, pour ma part, j'en avais retenu, et que parut d'abord confirmer la lecture d'ouvrages critiques de haute volée sur l'auteur du Pasticciaccio: ceux de Roscioni, de Fratnik ou de Manganaro, entre autres.

A priori, le haut degré d'abstraction qu'une réflexion sur Gadda semblait exiger m'arrangeait bien. Je venais d'achever et de soutenir, après cinq ans d'efforts, une thèse sur Dino Campana - qui ensuite deviendrait un livre-, pour laquelle j'avais apprêté et aguerri des outils de lecture assez nettement trans-historiques, visant à faire apparaître que l'enjeu crucial des Canti Orfici n'est pas dans un débat avec et dans l'histoire, mais dans une quête atemporelle de l'extase: même si le poète inscrit macroscopiquement, et sciemment, sa parole dans son temps, c'est toujours dans une tension de dépassement des formes de l'histoire, dans un élan - qui se découvre en chemin ou se savait d'avance vain - de délivrance de la prison des contingences et du temps. Dans mon ignorance de Gadda, j'entrevoyais que certaines de mes idées à propos de Campana allaient m'aider à entendre cet autre écrivain a-normal, Campana et Gadda - par-delà leurs différences voyantes - ayant pour trait commun d'être auteurs de textes dé-ments ou dé-lirants, au sens que l'étymologie peut redonner à ces adjectifs: des écrivains dont l'écriture, à partir de l'esprit (lat. mens) ou du sillon (lat. lira) de l'époque, s'en éloigne, s'en extrait sans retour, en quête d'autre chose qui n'est pas tout à fait d'ici.

Il y a une puissante inclination en chacun, une sorte de pente naturelle qui pousse à ramener l'inconnu au connu. Breton ou Artaud s'en sont parmi d'autres aperçus, pour regretter cette propension, et nous inviter à la combattre. C'est en effet souhaitable, si l'on veut que la pensée - et par conséquent la vie - ne se fige pas bientôt dans des formules - et par conséquent des comportements - assis une fois pour toutes. Mais quand on prépare un cours, ou même un livre, il n'est pas dit qu'on ait spontanément envie, à chaque fois, de s'exposer à l'inconfort qu'il y a, forcément, à ne pas faire cela que précisément on sait faire, à ne pas chercher à retrouver cela qui précisément nous est familier. Autrement dit, je ne demandais pas mieux que de lire Gadda comme j'avais lu - je crois avec profit, en tout cas pour moi - Campana: en ne considérant guère l'histoire à lui contemporaine que comme le visage particulier que prirent, pour lui, dans l'espace-temps qui fut le sien, les instances universelles, les vérités existentielles, les inquiétudes métaphysiques intemporelles à la méditation desquelles l'œuvre convie le lecteur.

Bien entendu, sur ce terrain, l'œuvre de Gadda me répondait assez généreusement. Pourtant, à mesure que j'avançais dans la lecture - que je 
voulais exhaustive - des cinq volumes que compte l'édition Garzanti de ses textes, je sentais grandir, contre mon gré, ma conscience de ce que cette approche ne suffisait pas; pire: qu'elle échouait à comprendre l'essentiel. Bientôt, la vision que je croyais claire dans ses grandes lignes devint absolument confuse, et je me retrouvais devant deux questions qui, sans doute, sont celles à quoi l'on arrive toujours quand on étudie un auteur: pourquoi choisit-il tels sujets ou domaines plutôt que tels autres; et pourquoi écrit-il de cette façon? Les réponses de type philosophique que j'avais cru avoir d'avance, celles que j'avais cru pouvoir dériver de mes recherches antérieures, ne me satisfaisaient plus. Il allait falloir regarder du côté de l'histoire, c'est-à-dire réfléchir sur ce que le texte gaddien doit en profondeur à une époque déterminée.

Quant aux territoires que Gadda explore, ce qui frappe aussitôt, c'est leur multiplicité. Il ne semble guère possible de résumer en quelques mots les domaines (et thèmes y afférents) de prédilection de cet écrivain (chose que l'on peut faire en revanche, sans trop de simplification, s'il s'agit de Calvino ou de Buzzati, de Pavese ou de Pirandello, de Sciascia ou de Primo Levi). Au mieux, il faut dresser une liste longue, et à première vue disparate: sociologie, linguistique et socio-linguistique, sciences exactes (mathématique, physique, hydraulique, mécanique...), peinture, philosophie, épistémologie, économie, histoire des idées, psychanalyse, littérature, histoire de l'art, histoire, politique, voilà - parmi d'autres - autant de secteurs dont Gadda s'est occupé, parfois avec une étonnante compétence, tantôt dans des textes qui se concentrent sur un champ, tantôt dans des livres où ils confluent et se mêlent tous.

Pour chacun de ces centres d'intérêt, on trouve sans trop de mal un ancrage précis dans l'histoire de Gadda, depuis la dimension du vécu personnel, jusqu'à l'échelle nationale, voire mondiale. Pour le plurilinguisme, par exemple, on invoquera ses périples à travers l'Italie, l'Europe, et jusqu'en Amérique du Sud, qui l'ont éveillé à la variété vertigineuse des parlers. Pour la curiosité scientifique, sa formation d'ingénieur. Pour l'histoire, son implication personnelle, en première ligne, dans des événements majeurs de son temps (la guerre, la montée du fascisme). Il semble possible de relier chaque domaine ou thème fréquenté par Gadda à un événement ou à une série d'événements ayant marqué sa vie, ou celle de sa famille, ou celle de sa classe sociale, ou celle d'une autre collectivité dont il se sentait partie prenante (les ingénieurs, les soldats, les écrivains, les Italiens, etc.). Avec, sous la multiplicité des sujets abordés, une préoccu- 
pation constante de connaissance, voire une ambition d'élucidation exhaustive et définitive. C'est ce qui permet de parler, à juste titre, de sa vocation encyclopédique, et amène à une question subsidiaire: pourquoi avoir ainsi voulu courir tous les lièvres à la fois?

De même que la raison peut rendre compte de la variété des thèmes traités en allant enquêter du côté de l'histoire, pour constater, comme je l'ai dit, que chacun correspond à un pan précis de la réalité vécue par Gadda, de même peut-elle s'expliquer son encyclopédisme, du moins le situer dans une loi générale: beaucoup d'écrivains eurent, ont et vraisemblablement auront longtemps encore cette même propension et cette même aptitude à s'occuper pertinemment de questions très diverses, pour les pétrir toutes et les brasser dans l'œuvre - même si cela finit le plus souvent par ne plus se déceler ${ }^{1}$. Gadda fait peut-être figure d'exception pour son époque et son pays, mais (c'est aussi l'avis de Manzotti) cela dénonce peut-être autant, ou davantage, certain provincialisme de la "haute culture» italienne du XX $X^{e}$ siècle, jusque dans les années 60 , que son absolue singularité - du moins si on le considère dans un panorama mondial, ou si on pense, même en se limitant à l'Italie, à des écrivains des siècles passés.

Gadda, dès 1924, était conscient de sa tentation d'exploiter la moindre de ses potentialités, lorsqu'il écrivait - à propos de ses «manières stylistiques" possibles, mais cela vaut par corollaire pour les registres thématiques: «Je regrette, $\mathrm{j}$ 'ai toujours regretté de renoncer à quelque chose qui me fût possible.» Et de poursuivre: "C'est cela mon mal» (RI, SVP, p. 396). Que cela soit un mal, c'est-à-dire une souffrance et une maladie, à l'heure où le savoir se fragmente en branches de plus en plus nombreuses et cloisonnées et où les repères qui garantissaient l'unicité du monde vacillent ou s'effondrent, certainement. C'est bien le mal de Gadda, en tout cas, que ce désir anachronique d'omniscience qui taraude son œuvre- et dont l'un des symptômes éminents est la tentative, évidemment vouée à l'échec, d'une narration omnidiégétique - et c'est par là qu'elle est le monument de complexité que l'on sait. Comme cela a été bien vu et bien dit par Manganaro (p. 83), Gadda aspire, contre le cours des choses qui désormais rend cela impossible, à renouer avec la tradition de l'homme universel, du savant qui réunit toutes les compétences, tel le

1. Comme on ne distingue plus entre eux les ingrédients lorsque la pâte est réussie. La littérature ne serait pas ce qu'elle est si elle ne se nourrissait pas d'autre chose que de littérature. Cioran en fait même un principe, avec l'excès jubilatoire qui fait son charme: «Ce qui rend les mauvais poètes plus mauvais encore, c'est qu'ils ne lisent que des poètes (comme les mauvais philosophes ne lisent que des philosophes), alors qu'ils tireraient un plus grand profit d'un livre de botanique ou de géologie. On ne s'enrichit qu’en fréquentant des disciplines éloignées de la sienne.» Cioran, p. 90-91. 
«poète-philosophe-soldat-mathématicien" qu'il déclare être dès avant ses premiers pas en littérature (GGP, SGF II, p. 753). Très tôt, une vocation au génie s'affirme chez lui, s'exprimant et se modelant dans une conception du savoir comme "somme de connaissances réelles", comme accumulation quantitative de tous les savoirs partiels, supposés solidement établis.

Pour récapituler ce premier point, on ne s'étonne plus ni de la grande variété des questions que Gadda a traitées, ni de son effort pour n'en (de son impuissance à en) privilégier franchement aucune, dès qu'on accepte de considérer qu'il se pense (se fantasme) en Omnilogue. À savoir: (a) qu'un aspect fondateur de son projet de vie et de carrière consiste dans le projet d'être grand - grand écrivain, grand homme, grand esprit; et (b) que sa façon de se représenter à lui-même la grandeur comporte, et donc lui impose de s'occuper de tout, de s'intéresser à tout, de n'être en reste dans aucun domaine du savoir, et de chercher à dire sur toute chose une vérité profonde, inédite, et, idéalement, définitive.

Je ne m'arrête pas aux motivations narcissiques qui s'attachent, si l'on veut, à cette sorte d'ambition. Après tout, qui écrirait encore, s'il mesurait, au moment même où il écrit, l'inanité absolue - au regard du génie d'autrui ou, plus anéantissant, sur l'échelle cosmique - de son effort pour laisser une trace? Il faut sans doute pour ouvrer une manière de foi aberrante en quelque chose d'absolu, et il n'est pas dit qu'il y ait solution de continuité ni degrés entre croire en une réalité divine, croire en l'éternité ou l'universalité de l'art, croire en son propre génie - au point de prendre la plume. Même athée convaincu, l'écrivain en train d'écrire est un croyant fervent.

Le fait est qu'on n'a de toute façon pas besoin de verser dans une psychologie de quatre sous pour comprendre l'ambition de tout dire sur tout qui aiguillonne Gadda, et en voir la pleine légitimité: "La considération d'un objet fini oblige notre esprit à reconnaitre l'existence de tout le connu, de tout le pensable, et autre chose encore.» (MM, SVP, p. 646). Vulgairement, c'est l'idée que tout est dans tout et réciproquement, que tout mène à tout et à n'importe quoi, idée qui, pour être banalement répandue, n'en est pas moins profondément irréfutable. Comprendre, com-prendre serait alors saisir le plus de donnés et de liens possibles entre les donnés dans les mailles du discours. D'où ce phrasé gaddien si caractéristique, hérissé de digressions et d'incises, de divagations et de notes, qui menace parfois d'enfler jusqu'au difforme-informe (le pasticciaccio des mots et des concepts, cuits et recuits à haute température). Et c'est un phrasé légitime, en tout cas épistémologiquement, car quoi que l'on dise, 
à n'importe quel propos, il n'y a pas de digression, jamais. La notion de digression n'a de sens que dans un cadre qui fixe arbitrairement (et toujours mensongèrement) un ordre sensé du discours, et définit ses marges et ses exclusions.

Si je parle d'une boule de billard, je peux parler du bois dont elle est faite, de l'arbre qu'on a coupé, de la forêt où il a poussé, du bûcheron qui l'a abattu, de sa femme, de ce qu'ils mangent, de l'histoire des jeux dans les lieux publics, de ce qu'on y buvait ou pas, de tout ce que la boule et la queue (de billard) peuvent évoquer, je reste toujours dans le sujet, je dis à chaque fois quelque chose qui se relie à ce sujet, sans que la notion de plus ou moins grande proximité entre ceci et cela ait encore un sens. Ce que le bûcheron a mangé avant d'abattre l'arbre a peut-être eu une incidence directe sur sa manière de travailler, de sorte que le bois a été plus dur ou moins dur qu'il ne l'eût été s'il avait mangé autre chose. Si le nez de Cléopâtre... Quoi que l'on dise autour de n'importe quel sujet est encore dans le sujet, car - c'est une autre idée-clef pour entendre Gadda - il n'y a pas de sujet (ni d'objet), c'est-à-dire pas de frontières nettes, stables, réelles, aux choses. Une chose n'est pas contenue dans son périmètre physique (ou abstrait, dans le cas d'une idée), mais elle est l'ensemble de ses relations avec les autres choses: avec toutes les autres choses. C'est le «mostruoso groviglio della totalità ", l'enchevêtrement monstrueux de la totalité, qui fait que quand je crois tirer un fil dans la bobine du réel, c'est le réel tout entier, et l'irréel avec, qui vient à moi.

Qu'on n'aille pas croire à des élucubrations, ni même à des spéculations oiseuses. Gadda est un homme formé à l'école des sciences dures, qui sait comment des objets très lointains, que séparent même parfois des distances inconcevables, ont entre eux des relations ( $\mathrm{f}$ )actuelles : la terre et le soleil dansent l'un par l'autre, l'œil et l'étoile se touchent. Et il pressent, plus qu'il ne peut encore le savoir, ce qu'on appellera bientôt la théorie du chaos et les systèmes dits "non linéaires", dans lesquels une variation infime (la chute d'un caillou) ici peut provoquer d'énormes bouleversements (l'engloutissement d'un village sous l'avalanche) dans un lointain ailleurs de l'espace-temps: "Si une libellule vole à Tokyo, elle enclenche une chaîne de réactions qui m'atteignent, moi.» (VM, SGF I, p. 654).

Il semblerait donc que j'aie aussi répondu en chemin à la deuxième question dont je suis parti : pourquoi Gadda écrit-il comme il écrit. C'est que son écriture est celle, pour le dire avec Montale, $\mathrm{d}^{\text {' }}$ un tradizionalista impazzito. » Un traditionaliste, c'est-à-dire quelqu'un qu'anime la conviction que l'érudition, l'entassement quantitatif des savoirs enregistrés et validés par la raison (entendons: par la raison dominante) peut, à la fin, 
permettre d'atteindre à une réponse dernière. Mais «impazzito", parce qu'évidemment, c'est une voie qui rend fou quiconque l'emprunte en croyant pouvoir arriver au bout. La folie, sinon de Gadda, de son écriture, c'est cette furie énumérative, digressive et assertive qui est la marque de son style, qui contraste si nettement avec la tranquillité avec laquelle Montale renonce, dès les Ossi di seppia, à la possibilité d'affirmer avec certitude, dès lors, écrira-t-il encore en 1966, que «si l'homme savait vraiment une chose il saurait vraiment toutes les choses.» (Montale, p. 2777). On voit que c'est au fond presque la même idée que chez Gadda (si connaître une chose équivaudrait à les connaitre toutes, c'est que chacune conduit aux autres et les contient), mais qui chez l'un se traduit par un renoncement axiomatique à tout comprendre, et même à comprendre quoi que ce soit (on ne saura jamais vraiment la moindre chose), chez l'autre par un projet éreintant et sans issue d'omniscience - fût-il, parfois, parodique: parfois, mais pas toujours.

Il semblerait, disais-je, que j'aie répondu à la question du style gaddien. Je pourrais donc m'arrêter là, si Gadda était pleinement sous le coup de l'illusion dont se démarque Montale: celle de pouvoir réellement connaître, ne serait-ce qu'une chose simple. Mais ce n'est pas le cas. Dès le tout début de sa carrière d'écriture, Gadda énonce lui aussi qu'on ne sait rien, même de quelque chose d'apparemment très familier: "On sait qu'en semant du blé, c'est du blé qui naît, mais on ne sait pas le pourquoi » (MM, SVP, p. 704); et que la science n'est qu'un trompe-l'œil:

Il faut reconnaître que le mystère du Péché Originel n'est pas un mystère plus grand que les mystères scientifiques. Et que la science physique et biologique est justement le domaine où les personnes honnêtes devraient dire "On n'en voit pas le fond" ou bien, si elles affectionnent plutôt les expressions du peuple, «On n'y comprend que dalle»; au lieu de dire toujours «On comprend» avec des airs magistraux, pour la raison qu'ils se sont donné des pseudo-causes pour expliquer l'inexplicable.» (MM, SVP, p. 716-717)

Voilà qu'alors la question du style gaddien se rouvre et se déplace: pourquoi, sachant ce qu'il sait, ne veut-il pas s'en faire une raison? Pourquoi ne veut-il pas s'avouer vaincu devant l'évidence pourtant reconnue de l'ignorance fondamentale? Pourquoi, autrement dit, s'attache-t-il à un mode d'écriture (c'est-à-dire aussi de pensée et de connaissance) qu'il savait d'emblée - très lucidement comme le montrent ces deux extraits et toute la Meditazione milanese - voué à l'échec et à l'enlisement? Pourquoi se condamne-t-il à cette "dysharmonie préétablie» dont les mécanismes ont été magnifiquement saisis par Roscioni, pourquoi engage-t-il son œuvre vers un désordre et un pasticcio certains, lui qui avouait dès 1916: "le pétrin [pasticcio] et le désordre m'anéantissent», "le désordre est mon continuel cauchemar» (GGP, SGF II, p. 570 et 597). 
À ce stade de ma réflexion sur Gadda, je comprenais donc toutes les raisons qu'il y a à écrire comme il le fait, des pages chahutées où les hiérarchies familières sont mises à mal, où sont contestées dans la pratique d'écriture les conventions qui organisent traditionnellement le récit, pour le rendre intelligible. Mais pourquoi n'écrire presque jamais autrement? Pourquoi travailler des années durant à enchevêtrer sa page, tandis que grandit en même temps le tourment de l'embrouillamini? Pourquoi écrire, par moments, du presque illisible, en contradiction flagrante avec l'ambition d'être reconnu du plus grand nombre possible? Décidément, les explications narratologiques, les légitimations épistémologiques ne me suffisaient plus. Ce serait renverser absurdement les choses, donner foi à un type erroné d'objectivité que de croire que la vie se conforme à des codes raisonnables et de nous réellement connaissables. La vie est ce qu'elle est (et nous ne savons pas quoi), et nous tentons de la saisir en énonçant des règles, qu'elle déborde toujours. De même - sauf exception clairement revendiquée, telle l'expérimentation oulipienne - une œuvre n'est pas le produit d'équations posées en amont; en tout cas, pas une œuvre de passion comme celle de Gadda. Il serait ridicule d'imaginer un Gadda passant sa vie à seulement illustrer une théorie gnoséologique, aussi fondée soit-elle, en écrivant des nouvelles et des romans, comme il serait ridicule de réduire la Recherche du temps perdu à une entreprise de défense et illustration de la syntaxe française. Le choix d'écrire en littérature démarque définitivement Proust du grammairien, Gadda de l'épistémologue. Quelque chose d'autre se joue, que l'inventaire des procédés n'épuise pas, et que l'on doit pouvoir entendre par les voies de la commune sensibilité, celles à travers lesquelles une œuvre nous atteint. Car, quelque complexe et docte et cérébrale qu'elle puisse être ou paraitre, l'œuvre en littérature est avant tout - ou si l'on préfere demeure après tout - œuvre de chair et d'émotion humaine. C'est ma conviction, autorisée par Gadda: «toute recherche est peine, toute création est douleur!» (VM, SGF I, p. 451). Que dans le cas de Gadda l'émotion, pour tenter de se dire, doive en passer (presque) toujours par le roncier de l'érudition ou de l'hyper-intellectualisation, c'est un fait, mais qui au fond ne nous apprend rien ni sur la nature de cette émotion, ni sur ses coulisses.

C'est ici que j'en vins à supposer l'existence d'une clef émotive aussi simple qu'est complexe l'édifice où elle permettrait d'entrer, dans l'intuition qu'il y eût justement un lien étroit, de proportion inverse, entre ceci et cela: vouloir tout dire, puisque c'est impossible et qu'on le sait, ne revient-il pas à vouloir taire quelque chose, à essayer de l'enfouir dans la prolixité? Telles ces personnes (chacun a pu en faire personnellement 
l'expérience, dans le rôle d'auditeur ou de locuteur) qui se montrent d'autant plus loquaces et minutieuses qu'elles ont, comme on dit, «quelque chose à cacher ".

Ayant imaginé qu'une telle clef puisse exister, je me mis à la chercher, en lisant et en relisant Gadda à l'écoute de ses silences - car il me semblait sensé d'envisager qu'un auteur qui énonce, dès le début de sa carrière, qu'il faut parler de tout à propos de n'importe quoi, m'en révélera davantage par ce qu'il exclut de son discours que par ce qu'il y inclut. Et un jour, à un moment précis, après de longues semaines d'obscurité et d'énervement contre moi-même et (pourquoi le taire) contre cet écrivain qui s'était soumis pour des raisons que je ne comprenais pas à la torture d'une telle écriture, et qui me l'infligeait sans que je puisse m'y soustraire, tenu que j'étais par mon devoir d'enseignant; ce jour-là une évidence m'a frappé et, je le dis au risque de sembler emphatique ou naif, tout s'est éclairé. J'étais, je m'en souviens, debout dans ma cuisine un livre de Gadda à la main, le volume intitulé Saggi Giornali Favole II de l'édition Isella. Ce détail est essentiel, car c'est ce volume qui contient le Giornale di guerra e di prigionia, le journal de tranchée et de captivité du sous-lieutenant, puis lieutenant Gadda, et que c'est ce journal de guerre qui m'a donné la clef que je cherchais.

Les pages que Gadda a écrites pendant la guerre entre 1915 et 1919 depuis son enrôlement jusqu'à un peu après son retour en Italie, après la captivité en Allemagne consécutive à la défaite de Caporetto - sont pénétrées d'un engouement guerrier plus ou moins crédible, mâtiné d'échos de la littérature de propagande, voire de circulaires de l'état-major: c'est le journal d'un officier qui se veut opiniâtrement ligio al dovere, et qui résout les conflits terribles entre devoir d'obéissance et sentiment de l'absurdité en s'imposant, à chaque fois, la nécessité de croire, d'obéir, de combattre. Mais quant aux silences dont j'étais à l'écoute, ils m'apparaissaient soudain plus bruyants que tout le reste: silence sur l'horreur, silence sur la mort, silence sur la terreur d'être exposé à une fin atroce, dont le soldat peut voir partout et sans cesse des exemples autour de lui, sur la personne de ses ennemis ou de ses camarades. Le discours sur la peur, singulièrement, sans être complètement éludé, détourne constamment l'attention des causes simples et évidentes (quoi de plus humain, de plus banal que la peur de mourir dans la tranchée?), pour invoquer des causes complexes, ou lointaines, en tout cas indépendantes du contexte de guerre. Telle cette "paralysie de la volition" que le soldat Gadda s'attribue comme une caractéristique intrinsèque de son être et que tantôt il fait remonter à son enfance douloureuse, tantôt met au compte de son tempérament excep- 
tionnellement sensible, voire de son statut malheureux de génie incompris; tels ces dérangements gastriques qui n'auraient d'origine que physiologique. N'était-il pas infiniment plus plausible d'imputer ces troubles du comportement (psychique ou intestinal) à la grande peur qui inhibe toute faculté ou, comme on dit, noue les tripes?

Admettre une explication de ce genre, la peur banale et commune du simple mortel sous les bombes, entrerait sans doute en flagrante contradiction avec l'idée que le combattant Gadda se fait de l'honneur du soldat et de la grandeur de la cause. Et parce qu'il la refuse pour lui-même, il la fustige avec une violence terrible quand il la reconnait chez l'autre, chez ces (et ses) soldats veules et incapables qui pensent à sauver leur peau, sur lesquels il appelle des châtiments exemplaires. On peut à bon droit dire que, quand Gadda écrit qu'il voudrait qu'on les égorgeât pour leur lâcheté, ce ne sont que des mots; mais on ne peut interdire au lecteur de se souvenir que ces mots sont écrits en temps de guerre, dans la tranchée, après que des circulaires de Cadorna ont promulgué, dès le lendemain de l'entrée en guerre du pays, le recours à la justice sommaire contre les "récalcitrants et les lâches", et donc, en somme, dans un contexte où l'on passe effectivement et immédiatement, quand l'honneur de la patrie est supposément en jeu, des mots aux faits. En bonne logique interne, les allusions à la mort sont rarissimes dans le Giornale: à l'exception d'un cas où il est question, très rapidement, de quelques corps, la seule description appuyée d'un mort concerne un soldat italien, tué par accident (et non au combat) en manipulant une grenade.

Mon hypothèse se voyait solidement étayée par le Giornale, qui me mettait face à l'évidence d'un triple déni : déni de la peur, déni de ses raisons triviales, déni, même, de la mort. J'entrepris de relire les textes que Gadda avait écrits ensuite à propos de la guerre, et constatai une semblable occultation ou partialité du propos: on peut parcourir de bout en bout les milliers de pages que Gadda a noircies, on verra que l'horreur effroyable de cette réalité-là demeure à la marge des récits et des réflexions, alors même qu'il n'a cessé de se redonner le thème de la guerre (celle qu'il avait vécue) comme objet de récit et de réflexion. Il n’y a que de très rares exceptions, qui à vrai dire confirment la règle: même quand est évoqué, dans les pages étonnantes de Manovre di artiglieria da campagna (MdF, RR I, p. 21-34), le chaos du combat du point de vue des soldats sous le feu, l'horreur subjective est mise en regard du spectacle objectif de la perfection mathématique des tirs commandés par le lieutenant d'artillerie l'ensemble de la nouvelle entretenant par ailleurs une confusion troublante entre manœuvres d'exercice et bataille pour de vrai. Pour le reste, 
pour peu qu'on ait quelque familiarité avec d'autres journaux de tranchée ou textes d'écrivains anciens combattants, ce qui frappe chez Gadda, c'est ce qu'il ne dit pas, et dont parlent Remarque, Barbusse, Genevoix, Dorgelès, Rebora, Lussu, Giono, Latzko, et même le peu pacifiste Jünger : les mutilations affreuses, les rats enragés de chair humaine, les très jeunes gens fauchés au premier feu, la discipline meurtrière, l'épouvante, l'alcool, les agonies, les morts absurdement inutiles...

Mais ce que je voulais comprendre, ce n'était pas la psychologie d'un individu nommé C.E. Gadda, qui ne m'intéresse qu'en tant qu'il a écrit des livres, et par les livres qu'il a écrits. L'hypothèse du déni n'était encore qu'un constat extérieur, et ne me disait rien sur la cohérence de cette écriture-ci. Il s'agissait maintenant de voir si on pouvait lui donner une place parmi les éléments qui conditionnent les modes mêmes de l'écriture gaddienne, et si oui, laquelle, et pour quel champ de pertinence: seulement les textes en rapport direct avec la guerre? d'autres aussi ? seulement une "période» de l'œuvre? ou toute la carrière, des premiers écrits aux derniers?

Quand on cherche à entendre un auteur, on désire spontanément, je crois, atteindre à une lecture unitaire, forger des clefs qui ouvrent non seulement un texte ici ou là, mais tous les textes qu'il a laissés. C'est bien ce désir qui s'avoue quand on parle, au singulier, de l'œuvre de tel ou tel, sans hésiter à réunir sous un même chapeau, dans l'hypothèse d'une possible interprétation globale, ses poèmes, ses essais, ses articles, ses romans, ses nouvelles, ses pièces de théâtre, et jusqu'à ses lettres plus ou moins privées. C'est sans doute l'effet, davantage que d'un excès de présomption critique, de cette intuition que Valéry met dans la bouche du narrateur de Monsieur Teste, déconcerté par le personnage éponyme: "L'incohérence d'un discours dépend de celui qui l'écoute. L'esprit me paraît ainsi fait qu'il ne peut être incohérent pour soi-même.»(Valéry, p. 27). Quand une hypothèse s'est avérée valable pour tout un pan de l'œuvre, on commence plus ou moins consciemment à l'essayer à l'œuvre entière - surtout si c'est une hypothèse "lourde" d'implications. On verra mieux ce que je veux dire si j'en énonce la forme inverse, dont le saugrenu saute aux yeux : viendrait-il à quiconque ayant lu Se questo è un nomo d'engager une étude de La chiave a stella en supposant d'abord, avant confrontation attentive au texte: ceci est un livre que l'auteur aurait pu écrire à l'identique même s'il n'avait pas connu Auschwitz? Que dans La chiave a stella il ne soit pas directement question des camps de la mort n'invalide pas, en soi, l'hypo- 
thèse initiale que l'expérience des camps soit fondatrice de ce texte-là aussi.

Sans doute cette aspiration à l'explication unitaire étend-elle ses effets bien au-delà des études littéraires, pour former un moteur de la recherche scientifique en général ${ }^{2}$, voire un principe de notre perception ordinaire du monde. Qu'on ne s'étonne donc pas du postulat que j'en vins à former, que voici : la guerre est l'alpha et l'oméga de tout ce que Gadda a écrit ${ }^{3}$; la guerre est à la fois le thème constant et l'impensable de son ouvre. $\mathrm{Ce}$ dont il ne cesse de s'occuper (même quand il s'occupe d'autre chose), sans pouvoir jamais s'en occuper vraiment (c'est pourquoi il s'occupe de tant d'autres choses). Ce qu'il voudrait par-dessus tout comprendre et (s')expliquer, mais qui reste l'impossible même de son écriture. Dès lors, sous réserve d'infirmations à venir, l'inachèvement propre à chaque entreprise de Gadda ne s'expliquait plus par les seules causes philosophiques et narratologiques, mais par l'impossibilité de clore le discours sur un objet d'emblée situé hors-champ, dont il ne peut/doit jamais être directement ou complètement question. Je m'aperçus alors que mon postulat m'avait sans doute été à mon insu suggéré par le titre que Gadda donne à un chapitre du Castello di Udine, le livre où en 1934 il revient (entre élégie et panégyrique national) sur son expérience de soldat: «impossibilité d'un journal de guerre.» (CUd, RRI, p. 134).

J'ai dit que mon enquête sur Gadda ne concerne pas la psychologie de l'homme. Il est pourtant toujours tentant de se livrer à un exercice de psychanalyse sauvage, propre à conforter les interprétations qu'un texte nous inspire ou, à l'inverse, à nous en suggérer pour saisir le texte. Dans le cas de Gadda, qui a si étroitement mêlé sa vie et son œuvre, les occasions de spé-

2. «La science est la tentative de faire correspondre la diversité chaotique de notre expérience sensible du monde à un système de pensée logiquement unifié.» Einstein, p. 77. Sur cette question, qu'il me soit permis de renvoyer à Mileschi, p. 51-53.

3. Qu'on veuille étendre le champ de pertinence d'un postulat donné à toute une œuvre ne veut pourtant pas dire qu'on considère que tous les autres postulats sont caducs. Il n'existe aucun postulat universel (sauf, peut-être, celui qui attribue toute chose à Dieu, ce qui nous exonère du même coup de chercher à rien comprendre). La physique le sait bien, qui ne se fonde certes pas sur un postulat unique, et qui parfois en invoque même plusieurs, contradictoires entre eux, pour observer un même phénomène. L'exemple de la lumière est connu: que le postulat qui dit «la lumière est une onde» permette de rendre compte de tels faits lumineux, quand tels autres ne s'expliquent que si l'on dit «la lumière est un faisceau de particules ", cela montre assez qu'un postulat ne nie jamais la possibilité et la validité des autres, même contraires. Si on a quelque réticence à l'entendre, c'est qu'on est pénétré de la conviction que, tout de même, il y a au fond une vérité, qui remplace et annule toutes les autres. Quand je dis qu'on peut lire tout Gadda selon l'axiome qui fait de la guerre son obsession et son silence centraux, je ne dis pas qu'on ne peut pas le lire tout autrement. On reste libre de poser par exemple, comme la critique je crois l'a fait souvent: quand Gadda parle de la guerre (ou de n'importe quoi), il parle en réalité de sa mère. En la matière, chacun choisit ses postulats, selon son goût et son entendement. Celui que je propose a au moins pour lui de n'avoir encore que guère été mis à l'épreuve des textes. 
culer sur les tenants et aboutissants de la fixation d'un complexe psychologique autour de la question de la guerre ne manquent pas: la mort "héroïque», aux commandes de son avion, du frère cadet et jalousé Enrico, qui rend inconsolablement déshonorante la défaite et la captivité de l'aîné; le sentiment de culpabilité d'avoir tiré un profit affectif de cette mort qui supprime un rival dans l'amour maternel; les origines hongroises de la mère, qui fait de la guerre contre l'ennemi austro-hongrois une guerre matricide (et fratricide, et suicide); l'incapacité à "tuer le père» (qui en 1915 était biologiquement déjà mort), dont le symptôme est la sujétion à l'autorité militaire et étatique... On peut poursuivre à loisir, mais l'intérêt de ces pistes me semble vite épuisé, d'une part parce que la psychanalyse suppose la collaboration consentante du sujet analysé (c'est pourquoi je l'ai dite, dans ce cas, sauvage), et d'autre part, et surtout, parce qu'invoquer les nœuds œdipiens et variantes de l'auteur revient à rabattre l'œuvre et les questions qu'elle pose dans le périmètre de la psychologie d'un individu particulier. Or, dans le cas de Gadda au moins ${ }^{4}$, c'est le mouvement inverse qu'il me semble décisif de décrire: comment, dans cet itinéraire apparemment si singulier, se révèlent de grands enjeux de la vie collective du XXe siècle.

Plutôt que de sonder l'inconscient de l'homme-auteur, j'entendais donc questionner ce qu'on appelle parfois l'inconscient du texte: lorsque je dis auteur, ou écrivain, ou Gadda, c'est par commodité, étant entendu que je ne m'occupe en réalité que des textes. La "psychologie de Gadda», si psychologie il y a, ne prétend en tout cas pas être celle de l'individu de chair et d'os qui s'appelait ainsi, mais celle de l'être fictif qu'il faudrait appeler "Gadda-tel-qu'en-ses-livres». C'est ce Gadda-ci qui, quand il parle et pense la guerre, le fait avec des outils conceptuels impropres à donner la mesure humaine (émotive, économique, historique, sociale, morale, etc.) de cette expérience: l'héroïsme, l'ordre, la raison, le devoir, la patrie sacrée, ses frontières intangibles, la haine obligée du «boche» (je cite), l'interdit jeté sur la peur, la splendeur de la cause, sa justesse, sa justice... Toutes ces entités ne seraient capables de rendre compte de - par exemple - l'effroi ou la jouissance qu'il y a à tuer qu'au moment où elles entreraient violemment en crise et seraient ou renoncées, ou reconnues

4. Je l'avoue dans le murmure des notes: je tends irrésistiblement à penser que ceci ne vaut pas que pour Gadda, mais pour tout écrivain qui a connu une expérience d'extrême violence collective. Comment, en effet, donner encore la priorité à la psychologie de l'individu, quand il s'agit de comprendre les effets d'un traumatisme à l'échelle d'un pays, d'un monde, de l'humanité? Dira-t-on à Primo Levi, de retour d'Auschwitz, que les causes les plus profondes de sa souffrance sont à chercher du côté d'un complexe d'CEdipe non résolu? 
comme les masques d'une pulsion assassine première toujours en quête d'alibis.

L'impropriété des termes dans lesquels Gadda essaie de penser et de dire la guerre trouve sans difficulté à se comprendre, dans le cadre du postulat que j'ai énoncé plus haut: lui qui excelle absolument à convoquer idées et méthodes inattendues et novatrices pour parler de bijoux, ou des doigts de pieds des saints sur un tableau de la Renaissance, se trouve à cours d'originalité s'il s'agit de la guerre. Au contraire, les notions qu'il invoque coïncident assez parfaitement avec celles qui règlent le discours de l'état-major, la voix de la propagande militaire, la rhétorique nationaliste, bientôt fasciste. Mais si elle est bridée dans l'explicite du discours sur la guerre, la créativité furieuse de Gadda n'est pas éteinte, et se reverse d'autant mieux ailleurs : ainsi peut-on dire que c'est d'avoir pratiqué d'un côté la langue univoque du pouvoir pour parler de la guerre que Gadda, de l'autre côté, fonde et développe avec une telle intelligence la critique de la «monolingua». On peut de même faire dériver la théorie du groviglio non directement de la fréquentation de Leibniz, mais de l'expérience du champ de bataille, qui pétrit et confond en un même hachis de causes et d'objets indémêlables les corps, la boue, les végétaux, les éclats de métal, et toute chose jusque là isolée dans ses frontières propres; et même dire que c'est parce qu'il fallait rapporter ce gâchis à quelque système de pensée logiquement unifié que Gadda, dans un second temps, se tourne vers Leibniz, à la recherche d'un soutien épistémologique. De même encore, on pourra dire que le conflit typiquement gaddien entre la crise du déterminisme et du roman-monde d'une part, le projet d'un roman manzonien d'autre part, représente (y compris au sens de présente à nouveau) cette contradiction fatale: de l'ordre parfait des tirs, de l'obéissance indéfectible, de la hiérarchie sans bavures sont sortis non l'harmonie, la beauté, la netteté: mais le chaos (concret et abstrait) de la guerre et de l'après-guerre. En ce sens, la dysharmonie à laquelle parvient Gadda est certainement, comme dit Roscioni, pré-établie, mais en tant que résultat inévitable de toute entreprise visant à imposer au monde et à la vie une géométrie inflexible.

Voici bientôt dix ans que je confronte aux textes mon postulat sur la guerre chez Gadda. S'il a subi quelques révisions en cours de route, son fondement a tenu bon. J'ai pu, grâce à lui, m'expliquer le surgissement, dans l'esprit d'Ingravallo plongeant ses yeux dans la gorge béante de Liliana, des monts Faiti et Cengio ( $Q P, R R I I$, p. 59), lieux métonyniques de la Grande Guerre, cités respectivement 21et 19 fois dans l'ensemble de l'œuvre gaddienne. J'ai pu comprendre pourquoi, dans son texte le plus 
abstrait, pour illustrer qu'il n'est nulle frontière véritable entre les objets apparemment séparés, Gadda prend tout soudain l'exemple d'un général en temps de guerre $(M M, S V P$, p. 670), pourtant le plus inadéquat qui se puisse pour donner l'idée de la fluidité du réel, de l'illégitimité des cloisonnements 5 . J'ai pu comprendre aussi - la liste n'est évidemment pas exhaustive - pourquoi la Première Guerre mondiale réapparait brusquement, dans la correspondance semi-privée que Gadda entretient depuis des années avec son cousin et confident Piero Gadda Conti, au lendemain de la Seconde, dans des lignes où se mêlent protestation de sa dignité et crainte d'être accusé. Et supposer aussi qu'il n'y a pas qu'une concomitance chronologique entre l'abandon définitif de la littérature en 1963, et la prise de conscience tardive dont témoignent ces déclarations, respectivement de 1961 et de 1967:

\footnotetext{
Donnez une carabine à un jeune homme, et au bout d'une heure il aura déjà tiré sur sa sœur, sur sa mère, sur le meilleur de ses amis.// Il faut vaincre le fascisme en nous-mêmes. (SGF I, p. 1181)

Comme faible et, peut-être, insuffisante excuse, qu'il soit tenu compte du fait que j'avais été emporté par des années terribles (comme tout le monde); que je n'avais pas eu la force de les affronter avec l'hérö̈sme nécessaire: que j'avais, en somme, manqué à tout, sur toute la ligne. J'étais déjà au bout de mes forces le 10 juin 1940, avant que la énième et la plus terrible guerre ne débouchât sur de nouvelles horreurs inouïes. Il y a beaucoup de choses que je voudrais te dire avant de m'éteindre, mais seulement oralement, comme en confession, pour n'être pas encore accusé de fautes qui ne sont pas des fautes et de crimes que je n'ai pas commis. Mes remords suffisent déjà à me crucifier. (Gadda Conti, p. 140)
}

Quelques remarques pour finir. Pour formuler, puis pour oser conduire de manière résolue cette lecture, je me suis trouvé aux prises avec notre accoutumance à penser la littérature comme ontologiquement séparée de l'histoire. Cette séparation n'est peut-être qu'une déclinaison particulière de la plus ancienne coupure entre l'âme et le corps. Le fait est qu'à l'école, on apprend à penser assis, immobilisé, dans la contrainte du corps. La littérature, comme toutes les activités supposées hautes de l'esprit, reléverait en dernière instance du cérébral, du spéculatif, du désincarné. Je ne parle pas en l'air: on trouve encore un peu vulgaire (variante: réducteur), ici ou là, de mêler aux nobles questions de la création (littéraire?) des considérations socio-historiques, politiques, surtout si elles amènent quelques millions de cadavres sur la table de la salle-à-penser. Gadda, d'une certaine façon, est l'auteur parfait pour ce genre d'approches, qui peuvent envisager la guerre dans la seule visée esthétique, en contradiction radicale avec

5. Que peut-il y avoir, en effet, de plus mortellement rigide que la hiérarchie militaire d'une armée en guerre? 


\section{Christophe Mileschi}

la poétique des auteurs, pour qui elle était bel et bien un objet historique: car on peut lire le Pasticciaccio, La cognizione, et bien d'autres textes, en ne sortant jamais du dédale des questions narratologiques, d'un structuraliste vertige a-historique, du palais des glaces métaphysique. Mais paradoxalement, Gadda est peut-être aussi l'auteur que l'on entend le moins bien en ne le lisant que de cette façon-là. C'est aujourd'hui ma conviction: le déni de légitimité que le texte de Gadda oppose à son lecteur - en l'écrasant d'érudition, en essayant de couvrir les marges du texte de digressions, de notes pléthoriques, comme l'a bien vu Manuela Bertone - est un défi, un appel, une supplique au lecteur: qu'il redonne à l'histoire et à la chair, à la matérialité physique des violences du siècle où l'auteur a vécu et écrit, leur centralité: celle qu'elles ne cessèrent de chercher en vain dans son œuvre ${ }^{6}$.

\section{Indications bibliographiques}

Pour les renvois aux textes de Gadda, j'utilise l'édition en cinq volumes établie par Dante Isella, Milan, coll. «I libri della spiga», Garzanti, 1989-1993.

La convention adoptée pour les volumes est la suivante:

Romanzi e racconti, vol. I : $R R I$

Romanzi e racconti, vol. II :

Saggi Giornali Favole e altri scritti, vol. I:

Saggi Giornali Favole e altri scritti, vol. II :

Scritti vari e postumi:

Et pour les œuvres:

Giornale di guerra e di prigionia:

Racconto italiano di ignoto del Novecento: $\quad R I$

Meditazione milanese: $\quad M M$

Il castello di Udine: $\quad$ CUd

La Madonna dei Filosofi: $\quad M d F$

Quer pasticciaccio brutto de via Merulana: $Q P$

IViaggi la morte: $\quad$ VM

\section{Autres ouvrages cités}

Bertone Manuela, Il romanzo come sistema. Molteplicità e differenza in C.E. Gadda, Rome, Editori Riuniti, 1993.

Bonifacino Giuseppe, Il groviglio delle parvenze. Studio su Carlo Emilio Gadda, Bari, Palomar, 2002.

6. Même la critique narratologique et métaphysique semble désormais vouloir en tenir compte. Dans un récent, intéressant et très documenté ouvrage relevant majoritairement de cette veine, l'auteur rappelle au passage l'historicisation nécessaire, et voulue par Gadda, de sa quête littéraire (Bonifacino, p. 75 $80)$. 
Cioran Emil, De l'inconvénient d'être né (1973), Paris, Folio Gallimard, 1988.

Einstein Albert, Conceptions scientifiques (1952), Paris, Flammarion, 1990.

Fratnik Marina, L'écriture détournée. Essai sur le texte narratif de C.E. Gadda, Turin, A. Meynier éd., 1990.

Gadda Conti Piero, Le confessioni di Carlo Emilio Gadda, Milan, Pan Editrice, 1974.

Manganaro Jean-Paul, Le baroque et l'ingénieur. Essai sur l'écriture de C.E. Gadda, Paris, Seuil, 1994.

Manzotti Emilio, "Gadda", in Letteratura italiana, dir. A. Asor Rosa, Vol. IV, Turin, Einaudi.

Mileschi Christophe, Dino Campana. Le mystique du chaos, Paris-Lausanne, L’Âge d'Homme, 1998.

Montale Eugenio, Il secondo mestiere. Prose, vol. II, Milan, Mondadori, 1996.

Roscioni Gian Carlo, La disarmonia prestabilita, Turin, Einaudi, 1969.

Valéry Paul, Monsieur Teste (1946), Paris, Gallimard, 1986. 